\title{
PIERRE BAYLE ET LE STATUT DE L'ATHÉISME SCEPTIQUE
}

Gianni Paganini* gianenrico.paganini@lett.unipmn.it

RESUMO Qual lugar é ocupado pela figura do " ateu cético » no esquema bayleano que se contrapõe tanto aos apologistas "latitudinários " (à maneira de Garasse) quanto aos teólogos mais "rigorosos 》 (à maneira de Voetius)? Bayle afirma, por um lado, uma "latitude" do ateísmo " especulativo» bem mais larga do que Voetius poderia aceitar, mas, por outro lado, faz valer um conceito preciso de divindade que apologistas como Garasse certamente julgariam excessivamente restritivo. Além disto, Bayle é bem consciente da diferença que separa toda forma de ateismo "dogmático » ou "afirmativo" do ateismo "cético". Mas é importante ressaltar que mesmo os céticos são considerados por Bayle como ateus "positivos 》 ou "ateus de especulação » uma vez que, para "ser não-Teísta ou Ateísta », não é necessário " afirmar que o Teísmo é falso, bastando considerá-lo como um problema ». Tudo isto compatibiliza-se bem com sua convicção que, da mesma forma que o teísmo, "há diferentes graus de Ateísmo ».

Palavras-chave ateísmo, Pierre Bayle, ceticismo, apologética

RÉSUMÉ Quelle place occupe la figure de l' " athée sceptique » dans le schéma baylien qui prend à contre-pied tant les apologistes "latitudinaires" (à la Garasse) que les théologiens plus "rigoureux» (à la Voetius) ? Bayle affirme d'une part une "latitude " de l'athéisme "spéculatif 》 beaucoup plus large que Voetius ne l'aurait accepté, mais d'autre part il fait valoir un

* Università del Piemonte, Vercelli. Recebido em 03/08/2009 e aceito em 22/09/2009.

KRITERION, Belo Horizonte, nº 120, Dez./2009, p. 391-406. 
concept précis de divinité que des apologistes comme Garasse auraient sans doute jugé trop restrictif. En outre, il est bien conscient de la différence qui sépare toute forme d'athéisme "dogmatique » ou " affirmatif» de l'athéisme " sceptique ". Mais il est important de remarquer que même les sceptiques sont considérés par Bayle comme des athées "positifs " ou des "Athéistes de spéculation » car, pour " être non Théiste, ou Athéiste », il n'est pas nécessaire " d'afirmer que le Théisme estfaux ; il sufit de le regarder comme un problême ». Tout cela cadre bien avec sa conviction que, comme pour le théisme, "il y a différents dégrez d'Athéisme».

Mots clés athéisme, Pierre Bayle, scepticisme, apologétique

La recherche contemporaine sur l'histoire de l'athéisme s'est progressivement éloignée d'un concept strict d'athéisme comme notion métaphysique (en tant que négation de l'existence d'une cause première, comme agent surnaturel) ${ }^{1}$ pour adopter une notion plus large d'incroyance (unbelief). C'est ainsi par exemple que l'Histoire de l'athéisme de Georges Minois réunit sous ce nom la plus large variété de phénomènes de non-conformisme religieux, d'hétérodoxie ou d'impiété. Comme l'a remarqué W. Schröder, qui se place dans une perspective tout à fait différente, cette approche 'élargie' a fini par convaincre aussi des historiens de la philosophie (ce que Minois n'est pas), comme les Néerlandais Wiep van Bunge et Michiel Wielema. ${ }^{2}$ D’une certaine manière, la version « latitudinaire » du concept d'athéisme a des antécédents à l'âge classique : il ne s'agit pas seulement de l'ouvrage du jésuite Père Garasse, La doctrine curieuse des beaux esprits de ce temps, ou pretendus tels (Paris 1623), où l'expression « athéisme » désigne une très grande variété d'oppositions à la doctrine catholique, depuis la simple critique de la doctrine de l'Eglise jusqu'au blasphème et à la négation véritable de Dieu. Cet ouvrage a l'allure typique des livres de controverse et se présente comme assez peu fiable, du point de vue historique, par sa tendance à faire flèche de tout bois dans l'intention d'attaquer ses adversaires. Une version plus sérieuse de cette

1 Concept que l'on retrouve encore par ex. dans le livre de Gaskin, J.C.A. Varieties of Unbelief from Epicurus to Sartre. New York-London, 1989. p. 2.

2 Schröder, W. « Hydra multiceps ou negatio existentia dei ? Garasse, Voetius et le concept d'athéisme », dans S. Taussig (éd.), La question de l'athéisme au dix-septième siècle. Turnhout : Brepols, 2004. p. 31 45. 
approche 'élargie' se trouve dans la conviction, très répandue parmi les théologiens de toute tendance, que la négation du gouvernement providentiel du monde par Dieu revient pour l'essentiel à une forme d'athéisme, bien que cette négation ne mette pas en question l'existence même de la divinité. C'est pour cette raison que les épicuriens (et plus tard les spinozistes) seront considérés comme des athées, même si ni les uns ni les autres ne nient l'existence d'une ou de plusieurs divinités, mais se limitent à abolir la notion de providence. ${ }^{3}$

Dans la recherche récente, l'utilisation d'un concept d'athéisme assez souple et nuancé a été proposée par Michael Hunter et David Wootton dans le recueil collectif qu'ils ont publié en 1992 sous le titre Atheism from the Reformation to the Enlightenment, mais ils avaient été précédés dans cette proposition d'une conception 'large' d'incroyance par le livre de F. Berriot sur Athées et athéismes au XVI siècle.

Le refus d'un concept métaphysique précis, c'est-à-dire étroit, d'athéisme est à présent contesté surtout par W. Schröder : celui-ci, dans son ouvrage sur la littérature philosophique clandestine Ursprünge des Atheismus, ${ }^{4}$ a proposé de revenir à une notion restreinte et philosophique d'athéisme, équivalente à la négation pure et simple de l'existence d'une cause première, identifiée avec Dieu. Par la suite, ${ }^{5}$ il est revenu sur les problèmes de terminologie et de méthodologie historique dans une intervention plus brève, en opposant à l' « usage polémique et imprécis » de Garasse un témoignage de la même époque beaucoup plus précis et rigoureux, celui de Voetius. Dans sa dissertation De atheismo (1639), ce théologien hollandais « recommende d'utiliser avec prudence le concept latitudinaire d'athéisme $»,{ }^{6}$ pour éviter les amalgames calomnieux si fréquents dans l'œuvre de Garasse. En effet, non seulement la classification systématique de Voetius comporte de très nombreuses sous-espèces d'athéisme, mais surtout elle propose une division préalable en deux catégories fondamentales : d'une part ce qu'on appelerait l'athéisme au sens large, qui comprend plusieurs formes (athéisme pratique, indirect, par conséquence, libertinisme, lucianisme, etc.) ; d'autre part, a contrario, une catégorie qui correspond précisément au concept métaphysique ou philosophique, l'« atheismus speculativus proprie dictus », qui désigne l'opinion de

3 Voir surtout Barth, Hans-Martin. Atheismus und Orthodoxie. Analysen und Modelle christilicher Apologetik im 17. Jahrhundert. Göttingen: Vandenhoeck und Ruprecht, 1971, passim.

4 Schröder, W. Ursprünge des Atheismus. Untersuchungen zur Metaphysik- und Religionskritik des 17. und 18. Jahrhunderts. Stuttgart : Frommann-Holzboog, 1998.

5 Schröder, W. art. cit. Voir aussi dans le même recueil notre article : Paganini, G. « Un athéisme d'ancien régime ? Pour une histoire de l'athéisme à part entière ", dans S. Taussig (éd.), La question de l'athéisme au dix-septième siècle. Turnhout : Brepols, 2004. p. 105-130.

6 Schröder, W. art. cit.. p. 36. 
ceux « qui sont profondément persuadés que Dieu n'existe pas ». En réalité, Voetius (comme la plupart des théologiens de son temps), juge qu'il n'existe pas « d'athées proprement dits », car cela reviendrait à nier l'existence d'une connaissance naturelle et innée de Dieu. Toutefois, et même s'il s'agit d'une sorte de classe vide, nous avons avec cet " athéisme spéculatif» un cadre conceptuel bien défini et qui correspond à un concept précis et philosophiquement exact d'athéisme. ${ }^{7}$

Cette brève introduction nous met donc en présence de deux controverses sur l'athéisme, celle du XVII et celle du XXI ${ }^{\mathrm{e}}$ siècle. En se référant à la classification de Voetius, Schröder s'est efforcé de dépasser l'une et l'autre en forgeant un concept « restreint» d'athéisme qui soit compatible tant avec l'apologétique de l'âge classique qu'avec nos exigences d'historiens de la philosophie (plutôt que de la culture ou de l'intellectual history au sens large du mot). Mais le cadre d'ensemble peut changer beaucoup dès que nous adoptons une pierre de touche différente de celle de Voetius. Nous verrons notamment que la classification de l'athéisme proposée par Bayle permet à la fois de répondre à des critères de rigueur conceptuelle et de décrire un phénomène qui a des contours et des gradations bien plus larges que celles considérées par Voetius (et par son interprète contemporain). Surtout, nous constaterons que le philosophe de Rotterdam a introduit dans son schéma de référence une notion enrichissante et 'moderne', celle de l'athéisme sceptique qui nous intéresse ici spécifiquement.

Mais avant de nous concentrer sur ce point précis, il convient de s'arrêter un instant aux motifs et aux contenus de l'attention dédiée par Bayle à l'athéisme. Nous le ferons en résumant les résultats de nos recherches antérieures et de quelques travaux plus récents.

On sait que la singulière réhabilitation de l'athéisme que Bayle prône en le jugeant préférable à l'idolâtrie ou à la superstition ne se fonde pas tant sur la figure de l'athée vertueux, lavé des habituelles accusations d'immoralité ou de débauche, mais surtout sur la reconnaissance d'un véritable contenu de pensée et de philosophie dont le négateur de Dieu serait le représentant, justement en tant qu' " athée de spéculation ». Contre la thèse de Voetius (qu'il connaît et cite), ${ }^{8}$ Bayle ne croit pas que la « classe » de ces athées soit nécessairement « vide»; au contraire, il affirme l'existence véritable de cette « aristocratie de

7 Tel est l'avis de Schröder, lequel constate pourtant l'« extrême marginalité de l'athéisme au début des temps modernes ", car il n'existerait qu'une demi-douzaine de textes athées (dans ce sens strict) qui aient été produits au dix-septième siècle.

8 R.Q.P. III, xiii (OD III p. 930b). Bayle se réfère notamment à cette affirmation de Voetius qu'il cite : “ Speculativi nulli sunt Athei qui certo persuasi sunt non esse Deum ». 
l'athéisme $»,{ }^{9}$ dont Spinoza est pour lui le représentant le plus accompli, tant par sa conduite morale irréprochable que par sa spéculation philosophique raffinée, bien qu'erronée aux yeux de Bayle : il est « l'athée de système » par excellence, à côté de ce Straton de Lampsaque, à moitié historique et à moitié fictif, dont Bayle reconstruit le système tout au long du dialogue imaginaire entre les deux jeunes Athéniens qui, dans la Continuation des Pensées diverses, sont à la recherche du naturalisme le plus cohérent.

Il faut dire tout d'abord que pour Bayle la définition de l'athéisme spéculatif ne se résume pas, comme l'indique Schröder, dans la simple énonciation d'une proposition « nucléaire » telle que celle-ci : « Dieu n'existe pas ». Tout au contraire, pour le philosophe de Rotterdam, l'athéisme est susceptible de degrés différents, dans un certain sens il est plus ou moins large, ou plus ou moins strict - du moins telle est la conclusion que l'on peut logiquement tirer du fait que le théisme, dont l'athéisme est la négation, est lui aussi susceptible d'interprétations différentes, qui font varier son contenu dans un sens plus ou moins élargi, plus ou moins restreint. ${ }^{10}$

Bayle se demande longuement, tant dans la Continuation des Pensées diverses que dans la Réponse aux questions d'un provincial, si les conceptions de la divinité ou, pour mieux dire, de la cause première, peuvent se ramener à un " centre de réunion » ou à un « centre d'unité ». Il ajoute immédiatement que l'athée partage avec le théiste l'idée de l'existence d'une cause métaphysique première, simplement il l'identifie avec la matière, la nature ou la nécessité, c'est-à-dire qu'il lui ôte tous les attributs personnels, moraux, surnaturels ou spirituels qui sont propres de la divinité, notamment au sens chrétien du mot. Dans un passage assez célèbre de la Continuation des Pensées diverses, en voulant infirmer la thèse du consensus gentium autour de l'idée de Dieu, Bayle ramène hardiment à une « dispute de mots» l'opposition entre athées et théistes. En effet, tant les uns que les autres reconnaissent l'existence d'une cause ou d'un principe premier, bien qu'ils se divisent sur le sens à lui donner. ${ }^{11}$

9 Mori, G. Bayle philosophe. Paris : Champion, 1999. p. 206 (pour la classification du phénomène de l'athéisme, cf. p. 214-15).

10 Nous avons analysé ces problèmes dans notre livre : Paganini, G. Analisi della fede e critica della ragione nella filsoofia di P. Bayle. Florence : La Nuova Italia, 1980. chap. VI, 3, p. 290-312.

11 Cf. C.P.D. xx (OD III p. 214a) : «Les Athées, sans en excepter un seul, signeront sincèrement avec tous les Orthodoxes cette thèse-ci : Il y a une cause premiere, universelle, éternelle, qui existe nécessairement, et qui doit être apellée Dieu. Tout est de plein pied jusque-là, personne ne fera incident sur les mots : et il n'y a point de philosophes qui fassent entrer plus souvent le nom de Dieu dans leurs systèmes que les Spinozistes ", que Bayle considère comme des athées. Dans une autre contexte (celui de la comparaison entre le matérialisme, soutenant un " dieu corporel », et le déisme), Bayle insinue le soupçon que tout le débat se réduit à une "disputes de mots ", s'agissant d'abord de s'entendre sur le sens qu'il faut donner aux mots en question (C.P.D., cxli, OD III p. 392b). Dans R.Q.P. II, cix (OD III p. 721b), le philosophe discute du problème du « centre d'unité » relativement aux conceptions polythéistes, et affronte dans les chapitres 
L'affirmation selon laquelle existe une cause première définit donc seulement les limites d'un genre dont, paradoxalement, théisme et athéisme constituent les espèces ; et la deuxième paraît beaucoup plus compréhensive, donc plus large et plus variée que la première. ${ }^{12}$ Dans ce contexte, Bayle se sert avec une certaine facilité d'une arme dialectique (l'accusation d'athéisme « implicite » ou « par conséquence », également admise par Voetius) et la dirige contre bon nombre de ces philosophies, surtout antiques, qui étaient traditionnellement considérées comme théistes. Quant aux tendances plus ou moins « latitudinaires » auxquelles il s'oppose, il les repousse du côté de l'athéisme (bien qu'implicite), précisément à cause de l'approche naturaliste du divin qu'elles révèlent constamment.

En outre, Bayle compte au nombre des athées "implicites » plusieurs philosophes qui, tout en admettant l'existence du divin, ne lui reconnaissent pas les caractères de personnalité, de liberté, de providence, de bienfaisance etc. ; en définitive, les « attributs moraux » de la divinité lui semblent beaucoup plus importants que ses attributs « métaphysiques » pour marquer la ligne de partage entre le théisme (au sens strict) et l'athéisme (au sens large). Le résultat peut nous sembler déconcertant, et il le fut pour la plupart des théologiens contemporains de Bayle, mais il faut reconnaître qu'il correspond à une ligne de pensée très cohérente chez lui : hormis le christianisme le plus rigoureux, le moins idolâtre et le moins superstitieux (une croyance, somme toute, assez restreinte, qui exclut non seulement les catholiques mais aussi les partisans d'une philosophie qui reconnaît à la nature en elle-même une certaine efficace), la grande majorité des conceptions philosophiques se trouve de facto englobée dans une sphère plus ou moins proche de l'athéisme, peu

suivants la question d'un dieu corporel, étendu, divisible afin de savoir s'il s'agit là d'une conception acceptable de la divinité pouvant ainsi être ramenée à un dénominateur commun générique du théisme.

12 Le passage C.P.D. xxi (OD III pp. 215b-217b) est fondamental sur ce point. On verra le début du chapitre : «On trouvera sans aucune peine un centre d'unité à l'opinion de tous les hommes, pourvu que l'on se contente de ce formulaire général, Dieu existe. Cette proposition n'excluant point nettement la pluralité des Dieux, et n'enfermant aucune action, auroit été acceptée par les Païens, aussi bien que par les Juifs, et n'auroit point déplu aux sectateurs d'Epicure ; et comme elle n'exprime point un être qui agisse librement, et avec une connoissance générale de toutes choses, les Athées les plus fiérez l'accepteroient ; car quel homme est assez bourru pour s'amuser à une dispute de mot dans une affaire comme celle-là ? " (ibid. p. 215b). Le concept est repris plus loin, au chap. xxviii où, discutant du « centre de réunion " auquel les latitudinaires réduisent les diverses formes de l'expérience religieuse, Bayle recourt à l'arme de la rétorsion, ou du passage à la limite: «Que si vous vous contentez d'un centre de reünion situé dans des idées abstraites, montez d'un degré plus haut, vous réunirez les Athées avec les Payens et avec les Juifs. Ils conviendront tous d'un être éternel qui est la cause de toutes choses, sauf à examiner après cela (car ils en feroient abstraction) s'il est libre, intelligent, etc.» (ibi p. 230b). En général, le problème, pour Bayle, concerne la détermination de la « nature » de Dieu, non l'existence d'une cause première. Comme annonce le titre marginal : « La question ne consiste point à établir s'il y a un Dieu mais à déterminer quelle est sa nature » et, dans le texte lui-même : « II n'y a rien de plus facile que de connoître qu'il y a un Dieu, si vous n'entendez par ce mot qu'une cause premiere et universelle » (C.P.D. xx, OD III p. 213b). 
importe qu'il soit déclaré (ce qui est très rare) ou qu'il reste implicite, comme dans la plupart des cas.

En ligne avec l'exigence d'articuler le contenu de la proposition qui porte sur l'existence d'une cause première, Bayle soutient donc la «Fausseté d'une idée de Divinité en général $»,{ }^{13}$ idée qui ne suffit pas par elle-même à identifier la base du théisme : ce qui implique, d'autre part, la fausseté d'une idée également vague et floue d'athéisme en général. Pour cette même raison, le philosophe de Rotterdam reviendra, dans une série de chapitres tout à fait parallèles de la Réponse aux questions d'un provincial, sur le sujet de la classification de l'athéisme et sur le problème de l'athéisme spéculatif; dans ces sectionslà, il empruntera encore l'expression « dispute de mots » ou « logomachie » pour définir le combat habituel du théisme et de l'athéisme en général. Pour lui, toute la partie se joue autour de la question de savoir de quel théisme ou de quel athéisme on parle. Comme dans la Continuation, Bayle y soutient que la dimension "personnelle » de la divinité constitue la vraie et authentique distinction entre théisme et athéisme. Encore une fois, ce n'est pas l'affirmation de l'existence d'une cause première qui fait la différence, mais la détermination de son « essence » ou de son « idée ": " C'est en ce sens-là que l'existence de Dieu est niée ou ignorée par les Athées, quoi que d'ailleurs ils ne nient pas ou n'ignorent pas que le monde n'ait une cause». ${ }^{14}$

Quelle place occupe la figure de 1' " athée sceptique» dans ce schéma complexe qui prend à contre-pied tant les apologistes « latitudinaires » (à la Garasse) que les théologiens plus "rigoureux » (à la Voetius) ? ${ }^{15}$ Bayle affirme d'une part une « latitude » de l'athéisme "spéculatif» beaucoup plus large que Voetius ne l'aurait accepté, mais d'autre part il fait valoir un concept précis de divinité que des apologistes comme Garasse auraient sans doute jugé trop restrictif.

En premier lieu, disons que Bayle est bien conscient de la différence qui sépare toute forme d'athéisme « dogmatique » ou « affirmatif » de l'athéisme « sceptique ». Le point de référence est de nouveau « la doctrine de Gisbert Voetius touchant l'athéisme spéculatif », dont il analyse les « logomachies », ${ }^{16}$

13 C.P.D. xxiv (OD III p. 221b). Sur l'influence que ces positions de Bayle eurent sur D. Hume, voir Paganini, G. «Hume, Bayle et les Dialogues Concerning Natural Religion », dans A. McKenna et G. Paganini (éds.), Pierre Bayle dans la République des Lettres. Paris : Champion, 2004. p. 527-567.

14 R.Q.P. III, xv (OD III p. 938a). II faut voir aussi tous les chapitres xiii-xv, pp. 931-43, qui abordent également la question de la facilité - ou non - de la connaissance de Dieu, sur laquelle Bayle observe : « A cela se rapporte ce que disent quelques-uns qu'il n'y a point de dispute sur l'existence de Dieu, mais seulement sur son essence » (XV p. 937b), niant que les deux questions puissent être séparées.

15 Remarquons que l'art. cit. de W. Schröder ne prend pas en considération la position de Bayle.

16 R.Q.P. III, xiii, p. 930b et sv. 
dans le but d'affirmer clairement l'existence réelle d'athées de « spéculation ». Tout d'abord, Bayle fait la différence entre l'athéisme « négatif » des peuples qui n'ont pas connu de Dieu par simple ignorance ou barbarie (comme les Antillais ou les Canadiens) et l'athéisme «positif» de ceux qui ont examiné à fond la question ( « ceux qui ont comparé ensemble le Théisme et l'Athéisme »). ${ }^{17}$ Il s'agit, dans ce deuxième cas, sans doute du petit groupe de philosophes anciens (Straton, Diagoras, Théodore) et modernes (Vanini, Spinoza et peu d'autres) qui font figure d'athées déclarés tant dans le Dictionnaire que dans les Euvres diverses. ${ }^{18}$ A son tour, l' " athéisme positif » se divise en deux « classes » qui le recouvrent disjonctivement: " Les uns - écrit Bayle - ne décident rien; les autres décident pour l'Athéisme. Ceux-là trouvant des dificultez tant du côté de la négative que du côté de l'afirmative, se tiennent en suspens ; ils sont Sceptiques ou Acataleptiques », selon qu'ils « continuent à examiner dans l'espérance de trouver enfin quelque certitude » (suivant l'acception «zététique » pour laquelle la recherche ne se termine jamais) ou qu'ils arrêtent leur recherche sur la négative ( « ils se persuadent que la question est impénétrable, et fort au dessus de leur esprit, et ils se fixent dans le doute »). En revanche, « [c]eux qui décident pour l'Athéisme, le font ou parce qu'ils le trouvent plus probable que le Théisme, ou parce qu'ils s'imaginent avoir des démonstrations $\gg{ }^{19}$ Spinoza en est l'exemple le plus évident.

Il est important de remarquer que les premiers, donc les sceptiques, sont considérés aussi par Bayle comme des athées "positifs » ou des «Athéistes de spéculation » car, pour « être non Théiste, ou Athéiste », il n'est pas nécessaire «d'afirmer que le Théisme est faux; il sufit de le regarder comme un problême ». Tout cela cadre bien avec sa conviction que, comme pour le théisme, « il y a différents dégrez d'Athéisme ». ${ }^{20}$

Certains vont beaucoup plus loin et finissent par regarder « l'autre parti » (la négation de Dieu) comme « une chose assez probable pour y pouvoir adhérer sans imprudence ». D'autres vont jusqu'au « comble de l'impiété » en affirmant que « le systême des Chretiens sur la nature de Dieu est impossible $»^{21}$ mais, insiste toujours Bayle, « les dégrez inférieurs à celui-là ne laissent pas d'être un Athéisme ». C'est ainsi que l'auteur de la Réponse introduit dans le débat une figure nouvelle, celle de l'athée sceptique, qui avait échappé à la

17 Bayle precise qu'il se sert de ce mot "théisme" « à l'imitation des Anglois, pour signifier en général la foi de l'existence divine » (ibid., p. 932n.).

18 Voir aussi la revue des « athées » contenue dans P.D. clxxiv (OD III p. 110 et sv.).

19 R.Q.P. III, xiii, p. 932a.

20 Ibid. p. 932 b.

21 R.Q.P. III, xiii, p. 932a. 
plupart des théologiens (à l'exception de David Derodon, à vrai dire beaucoup plus vague que Bayle sur cet argument). ${ }^{22} \mathrm{Il}$ décrit ces athées sceptiques comme ceux « qui s'éloignent également de l'afirmation et de la négation de ce grand article » (l'existence de Dieu) ${ }^{23}$

Il s'agit pour Bayle d'un phénomène moderne par excellence, même s'il plonge ses racines dans l'Antiquité, comme il résulte de la phrase attribuée à Protagoras : "Pour ce qui est des Dieux, je ne sai ni s'il y en a, ni s'il n'y en a point, ni ce qu'ils sont $»,{ }^{24}$ phrase qui peut être rapprochée de la formule sceptique ou mallon. Il s'agit aussi d'un phénomène qui selon Bayle est relativement majoritaire parmi ceux qui, élevés dans le christianisme, sont devenus incroyants, car « le plus grand nombre de ces gens-là ne font simplement que douter, sans en venir à l'afirmation réelle, qu'il n'y a point de Dieu », même si Bayle continue de penser qu'il doivent être rangés dans l'ensemble « des gens coupables de l'Athéisme positif et spéculatif $\gg .^{25}$

Mais le problème est justement de ne pas confondre l'agnosticisme sceptique des anciens, et des pyrrhoniens surtout, avec ce que Bayle envisage comme athéisme « sceptique » et qu'il attribue aux modernes. ${ }^{26}$ Comme Bayle le sait bien, le concept d'athéisme « sceptique » peut non seulement sembler une contradictio in adjecto, car il implique une position dogmatique, bien que de type négatif, mais surtout il est tout à fait étranger à la tradition pyrrhonienne, qui a toujours affiché un certain respect, du moins extérieur, pour les croyances religieuses de la cité, assimilées à cet ensemble d'orientations non dogmatiques comprenant les quatre aspects qui règlent la « vie ordinaire ». Dans ces dispositions, à côté du « guide de la nature », de la « nécessité des affections » et de l'enseignement des arts », on trouve aussi la « transmission des lois et des coutumes ", donc l'ensemble des croyances et des usages reli-

22 Voir notre communication présentée au Congrès des Lumières de Dublin (1999) : Paganini, G. « Avant La promenade du sceptique: Pyrrhonisme et clandestinité de Bayle à Diderot », dans G. Paganini, M. Benitez, J. Dybikowski (éds), Scepticisme, Clandestinité et Libre Pensée / Scepticism, Clandestinity and Free-Thinking. Paris : Champion, 2002. p. 17-46, surtout le $\S 1$ «Pierre Bayle et l'invention de l'athéisme sceptique », p. 17-23.

23 Avec une très grande finesse, E. Labrousse soutenait que Bayle parlait (implicitement) de son propre cas lorsqu'il ajoutait dans le passage qui suit immédiatement : «On ne prétend point parler de ces personnes qui se sentant incapables de satisfaire aux dificultez, tomberoient dans l'incrédulité si elles ne faisoient que suivre par une certaine route les suggestions de leur esprit, mais la conscience, les preuves de sentiment, les preuvess directes, le péril d'ofenser Dieu, le grand intérêt du salut les soûtiennent contre les objections les plus embroüillées ; et ainsi nonobstant les doutes qui se pourroient élever de tems en tems, ils se tiennent à l'afirmative » (R.Q.P., ibid., p. 932b-933a).

24 R.Q.P. III, xiii (OD III, p. 933a).

25 Ibid., p. 933a.

26 Ceux-ci ont “cessé d'affirmer mentalement qu'il y a un Dieu », même s'ils « s'abstien[nen]t de le nier mentalement », car les « objections contre l'athéisme ont dû lui paroître insolubles » (ibid., p. 933a). 
gieux. ${ }^{27}$ La polémique des sceptiques ne vise pas la religion populaire, mais plutôt les essais de certains penseurs, surtout stoïciens, pour la transformer en une sorte de théologie philosophique, et donc en un ensemble de « dogmes » que les « mythes » purs et simples ne veulent pas être. Très différents en cela des « dogmatiques » de tout genre, et donc aussi des « athées », les « sceptiques ", écrit Sextus, " à cause de l'équivalence des raisonnements opposés, affirment que les dieux n'existent pas davantage (ou mallon) qu'ils n'existent pas $» .{ }^{28}$ L'article « Pyrrhon » du Dictionnaire souligne bien cet aspect caractéristique du scepticisme ancien et l'oppose au scepticisme moderne. Bayle ne se contente pas de défendre la position de Pyrrhon contre les « mauvaises plaisanteries » ou plutôt les « impostures »d'Antigonus Carystius, en démontrant que le maître ancien n'était pas « fou » au point de refuser les apparences de la vie ordinaire ${ }^{29}$ de plus, l'article du Dictionnaire insiste sur la respectabilité de Pyrrhon, y compris sur le plan religieux. S'appuyant pour l'essentiel sur La vertu des Payens de La Mothe Le Vayer, Bayle soutient qu'on « doit le considérer comme Fondateur d'une grande Compagnie », et qu'il est par conséquent « sans doute recommandable en beaucoup de façons ». Qu'il se soit vu attribuer le titre de « Souverain Pontife » par les habitants de sa ville et que les Athéniens lui aient conféré leur citoyenneté le démontrent clairement. ${ }^{30}$

Le problème du rapport du scepticisme avec les croyances religieuses se pose évidemment en des termes fort différents dès lors que la religion soutient des prétentions doctrinales, et pas seulement morales ou civiques comme c'était le cas dans l'Antiquité. C'est alors que la coexistence entre le doute et la croyance se révèle difficile, même si les résultats peuvent être contradictoires, comme l'article « Pyrrhon » du Dictionnaire a l'honnêteté de reconnaître. Bayle y présente en effet deux thèses opposées : d'une part il affirme que le pyrrhonisme en tant qu' " art de disputer sur toutes choses » est " détesté » « avec raison" " dans les Ecoles de Théologie, où il tâche de puiser de nouvelles forces ", comme on le voit dans le célèbre dialogue des deux abbés philosophes dans la rem. B ; d'autre part, il soutient que, dans l'acception non moins célèbre de "pyrrhonisme chrétien», le scepticisme "peut avoir ses usages pour obliger l'homme par le sentiment de ses ténèbres, à implorer le secours d'en haut, et à se soumettre à l'autorité de la Foi ». ${ }^{31}$

27 Sextus Empiricus, Pyrrhonianae Hypotyposes, I, 237. Cf. aussi Adversus Physicos, I, 49.

28 Adversus Physicos, I, 59.

29 Bayle, P. Dictionnaire Historique et Critique, cinquième éd. Rotterdam-Amsterdam, 1740, «Pyrrhon », in corp. et rem. D.

30 Ibid., rem. D, t. III, p. 734b.

31 «Pyrrhon », in corp., t. III, p. 732-33. 
À l'égard du problème de la nature religieuse ou non du scepticisme, Bayle adopte ici la méthode pyrrhonienne et phénoméniste, qui oppose phénomène à phénomène, opinion à opinion. D'une part il représente dans toute sa portée édifiante la position 'officielle' de La Mothe, comme on peut la voir dans La Vertu des Payens, où le "pyrrhonisme » est décrit comme le "Parti le moins contraire au Christianisme ». Puis il fait un grand étalage de références très rassurantes à la théologie apophatique de Denys l'Aréopagite, à la critique paulinienne de la sagesse « humaine », à Calvin et à Pascal. Mais d'autre part et dans la même remarque, Bayle souligne que, selon d'autres « habiles gens » (La Placette et Gérard Vossius, qui cite à son tour Clément de Rome), «rien n'est plus opposé à la religion que le Pyrrhonisme », car celui-ci porterait «l'extinction totale, non seulement de la foi, mais de la raison ». La position de La Mothe Le Vayer est remarquable, car par les textes cités dans cette même remarque le libertin finit par jouer à la fois les deux rôles de la dispute. Il affirme en effet que «le système sceptique, fondé sur une naïve reconnoissance de l'ignorance humaine [est] le moins contraire de tous à nostre croyance », mais ailleurs il « exclut les Pyrrhoniens de la grace qu'il a faite à plusieurs anciens philosophes », en jugeant « désespéré le salut de Pyrrhon \& de tous ses disciples qui ont eu les mesmes sentimens que luy touchant la Divinité...», ${ }^{32}$ et cela même dans un ouvrage, comme La Vertu des Payens, qui avait été écrit dans le but explicite de 'sauver' la plupart des philosophes anciens de la condamnation janséniste, par le moyen du concept

32 Ces passages de La Mothe Le Vayer sont cités par P. Bayle, dans le Dictionnaire, loc. cit., p. 734a-b. Nous avions anticipé ces considérations sur le salut « désespéré » des sceptiques, d'après La Mothe Le Vayer, dans notre article «Avant La promenade du sceptique ... » cit., p. 23-31. J.-M. Gros a rejoint à peu près les mêmes conclusions à partir des mêmes textes (De la Vertu des payens, Bayle) dans son article «Le masque du "scepticisme chrétien" chez La Mothe Le Vayer ", Libertinage et philosophie au XVII siècle, 5 , 2001, p. 83-98. Nous ne partageons cependant pas les conclusions de J.-M. Gros, qui va jusqu'à à relativiser l'importance du scepticisme, soupçonnant qu'il soit «lui aussi, pour une partie au moins, une couverture », sinon un «masque », « un réfuge, un lieu de repli », car « la suspension du jugement débouche sur une acceptation extérieure des valeurs établies » ( La place du cynisme dans la philosophie libertine ", Libertinage et philosophie au XVII' siècle, 7, 2003, p. 121-139, spéc. p. 138-139). Plus proches de notre interprétation les conclusions qu'a rejoint Isabelle Moreau, « La Mothe Le Vayer, ou comment transformer un ouvrage de commande sur la grâce en défense et illustration des philosophes de l'Antiquité réputés athées ", dans Isabelle Moreau et Grégoire Holtz (éds.), Parler librement. La liberté de parole au tournant $d u$ XVI et $d u$ XVII siècle. Lyon : ENS Éditions, 2005. p. 159-170. Sur les rapports de Pierre Bayle avec la culture libertine, et notamment avec la philosophie de Le Vayer, voir, après notre livre (Paganini, G. Analisi della fede e critica della ragione nella filosofia di Pierre Bayle cit., p. 40-46, 85-89, 160-164, 257260), Giocanti, S. « Bayle et La Mothe Le Vayer », dans Antony McKenna et Gianni Paganini (éds.), Pierre Bayle dans la République des lettres cit., p. 243-263. Voir aussi les études de Lorenzo Bianchi («Pierre Bayle et le libertinage érudit », dans Hans Bots (éd.). Critique, savoir et érudition à la veille des Lumières. Le Dictionnaire de Pierre Bayle. Amsterdam-Maarssen : APA, 1998. p. 251-267), de Didier Foucault («Pierre Bayle et Vanini ", dans Hubert Bost et Philippe de Robert (éd.), Pierre Bayle, citoyen du monde. De l'enfant de Carla à l'auteur du Dictionnaire. Paris : Champion, 1999, p. 227-242) et de Sophie Gouverneur, «Bayle et l'écriture de Naudé », dans Pierre Bayle dans la République des Lettres cit., p. 265-286. 
de « foi implicite ». Au-delà du cas personnel de La Mothe, la conclusion de Bayle est d'une portée générale, concernant l'attitude religieuse en soi dans son rapport avec l'état d'esprit qui est produit par le scepticisme. En effet, les termes du problème regardent l' « opposition » entre la « Religion » et le «Pyrrhonisme» tout court, comme le rappelle le Dictionnaire un peu avant dans la remarque et comme Bayle l'avait déjà dit à la remarque précédente (B). À la différence de la «physique » et de la «politique », qui pourraient coexister avec le « doute » et qui se contentent d'un degré de " probabilité » sans prétendre à la certitude absolue, la théologie s'en méfie à bon droit : " c'est par rapport à cette divine Science que le Pyrrhonisme est dangereux ", car la religion, notamment le christianisme, « doit s'appuyer sur la certitude ; son but, ses effets, ses usages, tombent dès que la ferme persuasion de ses véritez est effacée de l'ame $» .^{33}$

Quant au jugement que Bayle porte dans son Dictionnaire sur la personne de Le Vayer, il est très équitable et soucieux, comme à son habitude, de ne pas spéculer sur ce qui relève de la conscience individuelle : ainsi Bayle le défend-il de l'accusation d'impiété ou de «n'avoir point de Religion », ce qui serait un « jugement téméraire », car - rappelle-t-il - « il y a une grande différence entre écrire librement ce qui se peut dire contre la foi, \& le croire très-véritable ». Mais d'autre part, lorsqu'il se réfère à l'œuvre juvénile la plus sulfureuse (précisément aux Dialogues d'Orasius Tubero), Bayle ne semble avoir plus de doutes, et même si la conduite personnelle de Le Vayer lui semble être celle d'un « sage » véritable, sur le modèle des anciens, il juge que «[c]ette régularité, cette austérité, cette sagesse, n'empêchèrent point qu'on ne soupçonnât qu'il n'avoit nulle Religion », surtout dans cet ouvragelà où il faisait paraître «trop de prévention pour la Sceptique, ou pour les principes des Pyrrhoniens », en définitive «beaucoup de libertinage » dans le sens intellectuel du mot. Malgré les apparences d'une apologie que cet article présente, il faut lire la défense de Bayle en filigrane, à la lumière des premières impressions de lecture qu'il avait librement manifestées dans sa correspondance (1674-1675), où il dit qu'il y a dans les Dialogues « bien de l'érudition, mais il y a encore plus d'impieté $\gg .{ }^{34}$

33 "Pyrrhon », rem B, p. 732a. Pour les jugements du Refuge sur cert art. "Pyrrhon », voir à présent Bost, Hubert. Pierre Bayle, Paris : Fayard, 2006. p. 431, 435.

34 «Vayer », in corp., t. IV, p. 408 : "C'étoit un homme d'une conduite réglée, semblable à celle des anciens Sages; un vrai Philosophe dans ses mœurs, qui méprisait même les plaisirs permis, \& qui aimoit passionément la vie de cabinet, \& à lire \& à composer des Livres. Cette régularité, cette austérité, cette sagesse, n'empêcherent point qu'on ne soupçonnât qu'il n'avoit nulle Religion. On se fondoit apparemment sur certains Dialogues qu'il avoit faits, \& qui parurent sous le nom d'Orasius Tubero, \& sur ce qu'en général il faisoit paroître dans ses Ouvrages trop de prévention pour la Sceptique, ou pour 
A la rigueur, on pourrait observer qu'après ce couple de thèses et antithèses sur les « mérites » religieux ou antireligieux du scepticisme contenus dans la rem. C de l'article Pyrrhon, la conclusion, surtout pour un sceptique, devrait être l'irrésolution, précisément dans l'esprit de l'isosthénie pyrrhonienne. Or c'est justement le concept sceptique de suspension du jugement (épochè) qui donne à Bayle la clef pour résoudre le problème complexe du rapport entre le scepticisme et la croyance, en faisant pencher la balance pyrrhonienne toute d'un côté, à savoir du côté de l'irréligion sceptique. Pour obtenir ce résultat, l'auteur du Dictionnaire se limite à rappeler un autre passage de La Vertu des Payens où La Mothe fait une distinction très claire entre l'athéisme de type « dogmatique » et celui qui est implicite dans le doute propre des Pyrrhoniens. Ceux-ci, remarque le libertin érudit, ne faisaient pas «profession d'athéisme, comme quelques-uns ont cru. On peut voir dans Sextus Empiricus qu'ils admettoient l'existence des Dieux comme les autres Philosophes, qu'ils leur rendoient le culte ordinaire, et qu'ils ne nioient pas la Providence ». Tout cela - nous l'avons déjà vu - appartient simplement au conformisme extérieur que les pyrrhoniens affichaient dans leur règle quadripartite. Mais cette foisci le jugement de La Mothe - évoqué par Bayle - va plus loin et pénètre la surface de l'attitude sceptique pour saisir ses motifs plus profonds : « Mais outre qu'ils [les Pyrrhoniens] ne se sont jamais determinez à reconnoistre une cause premiere, qui leur fist mépriser l'Idolatrie de leur tems, il est certain qu'ils n'ont rien cru de la Nature Divine qu'avec suspension d'esprit, ni rien confessé de tout ce que nous venons de dire qu'en doutant, et pour s'accom-

les principes des Pyrrhoniens. II est sûr qu'il y a beaucoup de libertinage dans les Dialogues d'Orasius Tubero : mais qui en voudroit conclure, que l'Auteur n'avoit point de Religion, se rendroit coupable d'un jugement téméraire ; car il y a une grande différence entre écrire librement ce qui se peut dire contre la foi, \& le croire très-véritable ». En note, Bayle cite une lettre de Guy Patin (13 juillet 1649), dans laquelle Le Vayer est décrit comme « autant Stoïque qu'homme du monde, homme qui veut être loué \& ne loue jamais personne, fantasque \& capricieux, \& soupçonné d'un vice d'esprit, dont étoient atteints Diagoras \& Protagoras " : il s'agit évidemment de l'accusation d'athéisme. Bayle connaissait les Dialogues dès sa jeunesse : voir sa lettre à Vincent Minutoli, du 12 juillet 1674 (Correspondance de Pierre Bayle, publiée et annotée par Elisabeth Labrousse, Antony McKenna et al., vol. I. Oxford : Voltaire Foundation, 1999. lettre 61, p. 290 : « J'ai lu quelques dialogues de Mr [de] La Mot[he Le Vayer], que l'on m'a asseuré n'avoir pas eté imprimez avec ses œuvres. Ils sont imprimez sous le nom d'Orasius Tubero, et ils contiennent des choses extremement hardies sur le fait de la religion et de l'existence de Dieu ") et la lettre à son frère Jacob, datée du 21 juillet 1675 (Correspondance, vol. II. Oxford : Voltaire Foundation, 2001. lettre 105, p. 250-251 : « Je leus à Rouen les Dialogues d'Orasius Tubero imprimés. Il y a bien de l'érudition, mais il y a encore plus d'impieté. Ce sont des coups de jeunesse, je l'avoüe, je ne sais pourtant pas si l'autheur s'en est repenti, car toute sa vie il à écrit à la deffense du pirrhonisme d'une maniere qui ne sentoit pas son ame fort devote. Je tiens Mr de La Mothe Le Vayer et Mr Naudé pour les 2 savans de ce siecle qui avoient le plus de lecture et l'esprit le plus epuré des sentimens populaires, mais parce qu'ils font trop les esprits forts, ils nous debitent bien souvent des doctrines qui ont de perilleuses consequences "). Encore dans une lettre à Des Maizeaux (3 avril 1705), Bayle raconte qu'il s'est servi des Dialogues d'Orasius Tubero (« que j'avois lûs autrefois d'un bout à l'autre ") pour remonter au passage célèbre où Bacon (dans ses Essais) démontre " que l'Idolatrie étoit pire que l'Athéisme » (OD IV, p. 852). 
moder seulement aux loix et aux coutumes de leur siecle, et du païs où ils vivoient ». En conclusion, la sentence de La Mothe Le Vayer est très sévère : n'ayant pas eu « la moindre lumiere de cette foi implicite », sur laquelle est fondée tout le dispositif visant à "sauver» les philosophes anciens dans la Vertu des Payens, "je ne voi nulle apparence de croire qu'aucun Sceptique ou Pyrrhonien de cette trempe ait pu éviter le chemin de l'Enfer $\gg .{ }^{35} \mathrm{C}$ 'est le dernier mot de La Mothe sur le problème du « salut» de Pyrrhon, mais surtout c'est aussi la conclusion de Bayle, car il ne commente visiblement pas cette sentence ni ne reprendra ailleurs une question si délicate. Comme on le voit, le problème capital est l'incompatibilité entre " la suspension d'esprit » (donc l'épochè) et la " certitude » ou la fermeté ("ferme persuasion ») que la religion et la théologie demandent. ${ }^{36}$ Dans la tension entre ces deux grandeurs incommensurables se mesure toute l'instabilité de la notion de «pyrrhonisme chrétien $\gg .^{37}$

Mais n'en restons pas là, car on pourrait subtiliser encore davantage le problème, en se demandant si la suspension pyrrhonienne ne représente en réalité un tiers parti entre le théisme et l'athéisme, plutôt qu'une forme d'athéisme " positif», comme pourtant le présente la Réponse. Dans l'esprit de ceux qui, au $\mathrm{XIX}^{\mathrm{e}}$ et au $\mathrm{XX}^{\mathrm{e}}$ siècles, ont présenté l'agnosticisme comme une position alternative tant à l'affirmation qu'à la négation de l'existence de Dieu, nous aurions dans l'épochè une troisième position. Pour exclure cette possibilité, il nous suffit de revenir à la Réponse aux questions d'un provincial. Bayle $\mathrm{y}$ distingue attentivement entre des gens qui sont devenus sceptiques après avoir été élevés dans le christianisme et d'autres, par ex. les lettrés chinois, qui apprennent « dès l'enfance un système de philosophie qui est un Athéisme tout pur ». Les premiers, lorsqu'ils cessent « d'être persuadés de l'existence de Dieu à cause de l'examen » qu'ils en font, finissent par imiter plutôt l'exemple de Protagoras avec son irrésolution sceptique ${ }^{38}$ que l'athéisme dogmatique des stratoniciens, des chinois ou des spinozistes. Mais ils n'en restent pas moins «Athéistes de spéculation », puisque la « différence spécifique » de leur classe d'appartenance, en contraste avec les « Théistes spéculatifs », serait

35 «Pyrrhon », rem. C, p. 734a-b.

36 Cf. supra, note $\mathrm{xx}$.

37 Pour une évaluation différente cf. José R. Maia Neto, The Christianization of Pyrrhonism. Scepticism and Faith in Pascal, Kierkegaard, and Shestov. Dordrecht: Kluwer, 1995. Nous avons repris le dossier de l'athéisme sceptique au XVII siècle, par rapport à La Mothe et à la discussion entre Descartes et Bourdin dans notre article : Paganini, G. «The Quarrel Over Ancient and Modern Scepticism : Some Reflections on Descartes and His Context », dans les Actes du Colloque de Los Angeles, The Legacies of Richard H. Popkin, ed. by Jeremy Popkin. Dordrecht: Springer, 2008. p. 173-94 et dans Skepsis. Le débat des modernes sur le scepticisme. Paris : Vrin, 2008 (coll. « De Pétrarque à Descartes »). p. 241-62.

38 R.Q.P., loc. cit., p. 933a (cité supra, p. xx, note $\mathrm{xx}$ ). 
de « n'être point persuadez de l'existence divine ». On pourrait conjecturer à la limite que Bayle est en train de décrire une expérience, ou au moins une tentation qu'il a vécue à la première personne : le plus souvent, remarque-t-il, au moins parmi les chrétiens, celui qui « a cessé d'afirmer mentalement qu'il y a un Dieu » « s'abstient de le nier mentalement; car les objections contre l'Athéisme lui ont dû paroître insolubles ». Et il n'est pas jusqu'à la métaphore de la balance ou de l'isosthénie pyrrhonienne que Bayle ne reprenne, bien que sous une forme différente : le sceptique moderne, écrit-il, " doit donc se tenir comme une piece de fer entre deux aimans de même force $» .{ }^{39}$ Remarquons que ce résultat final n'enfreint aucunement la règle sextienne de la suspension d'esprit et qu'il n'introduit aucun élément de l'extérieur pour forcer l'esprit dans un sens ou dans l'autre. La conclusion consiste plutôt dans le fait de maintenir la suspension et d'approfondir ce qu'elle signifie pour la conscience religieuse qui en serait affectée. Point n'est besoin ici de rappeler la dialectique compliquée des oppositions qui se manifestent pour Bayle, dans le cas de la théologie chrétienne, entre les notions à priori, en elles-mêmes acceptables, et les notions à posteriori, irréconciliables avec celles-là, opposition qui se reproduit maintes fois dans l'œuvre de Bayle, dès le Dictionnaire jusqu'au dernier ouvrage, les Entretiens de Maxime et de Thémiste. Cette situation, qui décrit exactement l'état d'esprit du chrétien assailli par les doutes sceptiques, peut bien correspondre à l'équilibre difficile de la suspension sceptique, mais à la lumière de ce que Bayle nous a expliqué sur l'opposition entre « épochè » et «persuasion» ou " certitude » religieuse, elle ne s'avère pas moins mortelle pour les exigences de la foi chrétienne.

Toutefois, notre but n'a pas été de «sonder les reins et le cœur» de l'homme Bayle (ce qu'il a lui-même reproché à Voetius), ${ }^{40}$ mais simplement de voir comment il a introduit dans le débat de son époque une figure intellectuelle relativement nouvelle, celle de l'athéisme sceptique. On assiste en effet, dans la Réponse, à la parution d'un « athée » " positif » et « spéculatif » de nouveau type, qui utilise le scepticisme comme un instrument critique et non dogmatique, et qui vise à miner des certitudes reçues plutôt qu'à bâtir des systèmes alternatifs.

39 R.Q.P. ibid., p. 932b-933a. Sur Bayle 'historien' de l'athéisme, voir les remarques de Kors, Alan Charles. Atheism in France, 1650-1729, vol. I. Princeton: Princeton University Press, 1990. p. 246-262, qui a souligné la tendance de Bayle à ne pas multiplier le nombre des philosophes explicitement athées ( $p$. 251 : «Bayle was always far more ready to exonerate than to convict individuals of atheism »). Mais à côté de cet athéisme « de fait », il y a aussi chez Bayle une sorte d'athéisme « de droit », qu'il a tendance plutôt à élargir et à étendre qu'à restreindre (cf. Paganini, G. Analisi della fede e critica della ragione nella filosofia di Pierre Bayle cit. p. 290-312).

40 R.Q.P., ibid. p. 932a. 
Il faut dire aussi que Bayle s'est ménagé une sortie du scepticisme, qui n'était pas l'issue de La Mothe Le Vayer. Il sortira de l'impasse en regardant du dehors, avec un véritable « regard éloigné », tant les deux partis en lutte (doute et croyance) que le principe de leur confrontation : ce qu'il fera en disant que l'âme «a sujet de se tirer de l'inquiétude » parce qu' " il n'y a jamais eu, \& il n'y aura jamais, qu'un petit nombre de gens, qui soient capables d'être trompez par les raisons des Sceptiques ». En effet, la « grace de Dieu dans les fidelles », la « force de l'éducation » dans les autres hommes, et aussi «l'ignorance» ou «le penchant naturel à décider» (ce que E. D. James a appelé «a provocative trio ») ${ }^{41}$ tranchent le nœud, bien qu'ils ne puissent pas le résoudre, en faisant ainsi « un bouclier impénétrable aux traits des Pyrrhoniens, quoique cette Secte s'imagine qu'elle est aujourd'hui plus redoutable qu'elle n'étoit anciennement $\gg .{ }^{42}$ En déplaçant l'enjeu de la dispute de la simple dialectique rationnelle au niveau de l'anthropologie, où convergent, toutes différences confondues quant à leurs effets, des ressorts surnaturels (la grâce) et d'autres mobiles tout à fait naturels et même trop humains (l'éducation, l'ignorance, le penchant instinctif), Bayle imprime un tournant décisif à la dispute, en préfigurant le problème huméen, à savoir si le scepticisme est une attitude humainement soutenable, ou bien s'il n'est pas plutôt continuellement dépassé par les impulsions « naturelles ».

41 J'emprunte cette expression à James, E. D. «Scepticism and fideism in Bayle's Dictionnaire », French Studies 16 (1962) : 307-323, p. 316 notamment. Cet article a provoqué une discussion qui mériterait d'être reprise. Voir Mason, Haydn T. « Pierre Bayle's religious views », ibid. 17 (1963): 205-217; Bracken, Harry M. "Bayle not a sceptic ? ", Journal of the History of Ideas 25 (1964): 169-180.

42 «Pyrrhon », rem. B, p. 732a. Sur la « mise en accusation » de la foi humaine dans le long parcours qui conduit de Montaigne à Bayle, voir les remarques convaincantes d'Antony McKenna, " "lls croient, ou ils croient croire" : réflexions sur la foi chez Montaigne et chez Bayle ", Bulletin de l'Association des Amis de Montaigne, janvier 1999. 